\title{
Identificación de capacidades digitales en estudiantes y docentes en educación media superior mexicana
}

\author{
Javier Tarango \\ Universidad Autónoma de Chihuahua, México \\ Fidel González-Quiñones \\ Universidad Autónoma de Chihuahua, México \\ Elsa Ivonne Morales-Ángel \\ Colegio de Bachilleres del Estado de Chihuahua, México
}

ORIGINAL

\begin{abstract}
Resumen
Objetivo. Describe las capacidades de estudiantes y docentes de Educación Media Superior (EMS) con el fin de identificar sus habilidades para el uso efectivo de las TIC, niveles de alfabetización informacional, habilidades de comunicación usando las tecnologías y competencias en la generación de contenidos.

Método. Es una investigación tipo cuantitativa (basada en la medición numérica y el análisis estadístico) y aplicada (propone establecer patrones de comportamiento), basada en un diseño de investigación no experimenal transeccional descriptivo, con un modo de campo empírico recolectando información a través de un cuestionario aplicando muestreos aleatorios de estudiantes y docentes de bachillerato general (no especializado o técnico).

Resultados. Se observa una variabilidad de tendencias en los propósitos en el uso de las TIC, tanto en docentes como en estudiantes, en la categoría de niveles bajos, al favorecer en términos generales a los primeros, poniendo de manifiesto la necesidad de acrecentar tales condiciones en razón de mejorar los procesos educativos y sociales a través del uso de las tecnologías. Conclusiones. Los resultados obtenidos favorecen a los docentes, ya que el estudio diagnóstico no se basa sólo en horas de conexión sino en la capacidad de utilizar el tiempo para realizar actividades productivas. Se propone establecer mecanismos de monitoreo para determinar el uso de las tecnologías en aspectos productivos y no sólamente en cuestiones lúdicas, así como en medir formas de impacto positivo en la competitividad de los sujetos y de la comunidades escolares.
\end{abstract}

Palabras clave:

Alfabetización informacional; Alfabetización mediática; Brecha digital; Capacidades digitales; Docentes universitarios; Estudiantes universitarios.

\section{Identification of digital capacities in students and teachers in Mexican high school education}

Abstract

Objective. Describes the capacities of students and teachers of High School Education (EMS) in order to identify their skills for the effective use of ICT, information literacy levels, communication skills using technologies and competencies in the generation of content.

Method. It is a quantitative type research (based on numerical measurement and statistical analysis) and applied (proposes to establish behavior patterns), based on a descriptive transectional non-experimental research design, with an empirical field mode collecting information through a questionnaire applying random sampling of students and teachers of high school general (non-specialized or technical).

Results. There is a variability of tendencies in the use of ICT, both in teachers and students, in the category of low levels, favoring the former in general terms, highlighting the need to increase such conditions in reason to improve educational and social processes through the use of technologies.

Conclusions. The results obtained favor the teachers, since the diagnostic study is not based only on hours of connection but on the capacity to use the time to carry out productive activities. It is proposed to establish monitoring mechanisms to determine the use of technologies in productive aspects and not only in ludic issues, as well as in measuring ways of positive impact on the competitiveness of subjects and school communities

Keywords:

Digital capabilities; Digital divide; Information literacy; Media literacy; University students; University teachers. 


\section{Introducción}

En el caso particular de México, el sistema educativo en los niveles de preescolar a bachillerato, experimentaron recientemente una reforma sustancial a través de la propuesta de un nuevo modelo educativo que fundamentalmente se caracteriza por tres aspectos generales (Gómez Collado, 2017): (i) integración de un modelo pedagógico que fomente en los estudiantes el pensamiento y el análisis, buscando desarrollar su capacidad de investigación, síntesis y estructura de conocimiento; (ii) imponer la enseñanza de una segunda lengua (inglés) desde temprana edad; y (iii) dotar de mayor autonomía a las escuelas.

Tales propuestas han generado una serie de inquietudes polémicas en todos sus actores, al grado que ha afectado múltiples condiciones estructurales, sociales y sindicales, sin embargo, se obvian diversos aspectos que, desde otras perspectivas, inhiben el verdadero cambio educativo, tales como: las desigualdades en el acceso y en las condiciones de escolarización, la calidad de lo que se aprende, generar sistemas en torno a las escuelas y el aprendizaje y todos los temas transversales probables, entre los que merecen especial atención las tecnologías y el papel que juega el docente en todo ello (Ramírez Raymundo, 2015), por tanto, se observa que esta clase de propuestas aventuradas no analizan las condiciones prospectivas que puedan llevar a una planeación adecuada y efectiva (McGinn, Rivera y Castellanos, 2014) y se carece de un análisis del cambio de la continuidad de políticas educativas con miras a mejorar la capacidad de intervención gubernamental (Del Castillo-Alemán, 2012).

El problema en cuestión no es generalizar el análisis de todos los factores, que a la luz de la literatura publicada pudieran identificar las carencias de las reformas educativas en México, sino en identificar uno en particular (las capacidades digitales) desde la perspectiva de sus propios actores: los estudiantes (como los principales beneficiados de los cambios) y los docentes (como los responsables de la implantación de los cambios, quienes enfrentan la posibilidad de ser superados y luchan por mantener a la innovación educativa dentro de las aulas). Preocupa entonces, el rol del docente en la era digital, ante el desarrollo imparable de las tecnologías y la democratización en su uso, siendo esta la condición que más ha transformado el contexto del proceso educativo, que, ante la necesidad de atender estudiantes nativos digitales los docentes dejaron de ser los guardianes del conocimiento (Viñals Blanco y Cuenca Amigo, 2016).

\section{Docentes, estudiantes y ambientes educativos: interdependencia para generar capacidades digitales}

Los entornos educativos y sus actores están condicionadas a los siguientes aspectos que definen su comportamiento actual:

a) En la actualidad, el sistema educativo enfrenta un gran desafío en su modelo educativo vigente, es de prioridad el implementar cada vez más programas que ayuden a entender y usar adecuadamente la tecnología, con la finalidad de que sea una herramienta útil, mejorando así el aprendizaje en los estudiantes, creando competencias y aprendizajes esperados, utilizando de una manera óptima las TIC (Zavala, Muñoz y Lozano, 2016).

b) El avance acelerado en la tecnología, ha implicado varios cambios y enfoques distintos en diversos sectores, y la educación juega un papel muy importante en el desarrollo de las tendencias digitales, constituyendo en gran medida una opción para que las instituciones educativas mejoren los procesos de enseñanza y, por ende, la calidad educativa crezca (Ceballos, 2013).

c) Si bien el internet ha impactado a nivel mundial, al ser humano en todos los aspectos, hoy en día, el acceso a diferentes bienes y servicios, de entretenimiento, socioculturales, e infinidad de plataformas amigables, los tenemos a un clic de distancia. Es increíble cómo se ha transformado la capacidad de respuesta y la variedad de opciones que tenemos al alcance de nuestras manos (Ugalde Sánchez y González Cabrera, 2016).

d) En muchos países la inversión en las TIC es insuficiente, lo que conlleva a generar una población con una pobre capacidad en el uso de la tecnología, resultado de una condición similar en el sistema educativo que inhabilita a la sociedad para hacer un uso efectivo de la misma. El integrar tecnología en las aulas, va más allá del simple uso de la computadora. Para que sea efectiva se necesita profundizar en los procesos de aprendizaje y en las necesidades de cada sector (López Meneses y Esteban Ibáñez, 2008; López, 2012; Marcelo, 2013).

La integración de las TIC en los sistemas educativos conlleva amplias dificultades, generalmente asociadas con (Fernández Cruz y Fernández Díaz, 2016; Colás Bravo, De Pablos Pons y Ballesta Pagán, 2018): (i) la falta de preparación de los docentes para introducirlas a su práctica habitual; (ii) las instituciones educativas regularmente 
no disponen de una propuesta acordada al interior de las mismas como proyecto propio; (iii) se carece de elementos de innovación para cambiar las clases tradicionales; y (iv) los estudiantes se sienten fuera de contexto, especialmente cuando ya se tiene algún avance en conocimientos tecnológicos.

Estudiar el bachillerato en México suele tener un significado diferente a otros países. Según la visión sociológica de Weiss Horz (2018) en una investigación con adolescentes mexicanos identificó que sus prioridades son: (i) el pase automático a la educación superior en el bachillerato general y la tendencia a la terminación de su trayectoria escolar al estudiar bachillerato tecnológico; (ii) la posibilidad de continuar estudiando y trabajando, especialmente en aquellos que observan una condición económica baja; y (iii) la posibilidad de adquirir un empleo formal a partir de los conocimientos adquiridos en la EMS.

En cuanto a la preparación de los docentes, las recientes normativas sobre la elaboración de planes de estudio y ambientes educativos en general aluden a la importancia de las tecnologías en la educación desde la educación básica, en donde se incluyen competencias sobre recepción mediática y menciones especiales en el ámbito de las TIC, sin embargo, se ignora incluir descriptores específicos de manejo de nuevas tecnologías, competencias en el ámbito de recepción crítica, alfabetización mediática e incluso producción de nuevos medios (Aguaded-Gómez, 2009). Esto representa que existe un problema de analfabetismo digital, lo cual se convierte en un obstáculo para generar ambientes educativos vinculados con las tecnologías, situación que sucede especialmente en aquellos profesores entre los 45-65 años de edad (Valencia-Altamirano, Topón Gualotuña y Pérez Fabar, 2016). Incluso, el uso de las TIC por los docentes sigue siendo tradicional, ignorando evoluciones y bondades del Internet 2.0 (Zempoalteca Durán, Barragán López, González Martínez y Guzmán Flores, 2017; Aguiar, Velázquez y Aguilar, 2019).

Para lograr un cambio en la formación de docentes en relación con sus capacidades digitales y la proyección que se haga a la realidad educativa, representa la posibilidad de un acercamiento a dos condiciones especiales, consecuencia una de la otra (Antúnez Sánchez, Morales Salas y Ortiz Ortiz, 2019): (i) la infotecnología (disciplina clave para lograr mayor cultura tecnológica a través del uso de herramientas de búsqueda, revisión y procesamiento de información científica; y (ii) la cultura informacional (vinculada con aquellos hábitos, costumbres y habilidades que la persona desarrolla después de un proceso educativo formal, familiar y social basada en cultura, información alfabetización y tecnologías. Entonces, la relación entre la alfabetización informacional y competencia digital están ampliamente relacionados, en donde incluso se ha identificado que los estudiantes que estudian para el magisterio son poco asiduos al uso de los recursos tecnológicos y audiovisuales, además, carecen de un vacío formativo (Moreno Rodríguez, Gabarda Méndez y Rodríguez Martín, 2018), por lo que no se puede esperar que cuando se incorporen al mercado laboral muestren un vínculo suficiente con el tema de interés en las TIC en educación.

Al considerar al profesorado como un actor fundamental en el desarrollo de las competencias digitales, se propone establecer mecanismos de medida y certificación en el tema, ya que esta competencia es clave en el desempeño de su profesión, desgraciadamente muchos de los estudios realizados corresponden a la medición de la autopercepción, con lo cual no se puede llegar a procesos de certificación (Hernández Ramos y Torrijos Fincias, 2018; Durán Cuatrero, Prendes Espinosa y Gutiérrez Porlán, 2019). Aunque los docentes y estudiantes puedan observar diferencias o no en la competencia digital, es importante considerar que finalmente el profesorado tiene mayores posibilidades de capacitación no sólo en el uso de las TIC, sino, además, en relación a la mejora de los procesos de generación de conocimiento (Paredes-Parada, 2019), al llegar a la condición de que, cuando el profesorado acceda a las TIC al grado de lograr su apropiación, será cuando se logre incluso a cambiar su propio perfil profesional (Tejeda Fernández y Pozos Pérez, 2018). La formación de los docentes debe considerar tres aspectos críticos (Castañeda, Esteve y Adell, 2018): (i) partir explícitamente de un modelo de acción docente dentro y fuera del aula, con compromiso social y político, así como la contribución de la escuela al desarrollo de la comunidad; (ii) generar competencias basadas en la complejidad, con lo cual se construya la identidad del docente; y (iii) evitar el mero instrumentalismo de las TIC, como herramienta neutra de valores en relación con la sociedad.

\section{Metodología}

La investigación observa las siguientes características:

a) Tipo cuantitativa ya que utiliza la recolección de datos con base en la medición numérica y el análisis estadístico.

b) Tipo aplicada, ya que propone establecer patrones de comportamiento y probar teorías en base a un modelo establecido previamente.

c) El carácter de la investigación fue no experimental porque no se realizó una manipulación deliberada de variables y solo se recabaron datos para ser analizados después.

d) La investigación es de tipo descriptiva pues busca especificar propiedades, características y rasgos importantes del fenómeno de las capacidades digitales en estudiantes y docentes universitarios describiendo tenden- 
cias en la población.

e) El diseño de la investigación fue no experimental transeccional descriptivo porque la aplicación de encuestas se realizó en un periodo específico de tiempo (enero-junio, 2018).

f) El modo de campo fue empírico, aplicando los cuestionarios en el ambiente donde se desempeñan, tanto estudiantes y docentes universitarios de una institución de educación media superior que ofrece bachillerato general (no especializado o técnico), basado en la siguiente muestra: (i) población de 2,584 estudiantes, con un muestreo probabilístico por conveniencia de 331; y (ii) población de 121 docentes, de los cuales 58 accedieron a responder el cuestionario.

Los criterios de evaluación según el cuestionario se basan en cuatro dimensiones de análisis según el modelo de Gigler (2011) y Gigler y Bailur (2014), adaptado por González-Quiñones, Tarango y Fierro-Ramírez (2018) y posteriormente, publicado como un modelo de medición validado (González-Quiñones, Tarango y Vallanueva Ledezma, 2019), tal como se expresa en la Figura 1 y posteriormente se describe:

Figura 1. Modelo de Diagnóstico de Capacidades Digitales (DICADI)

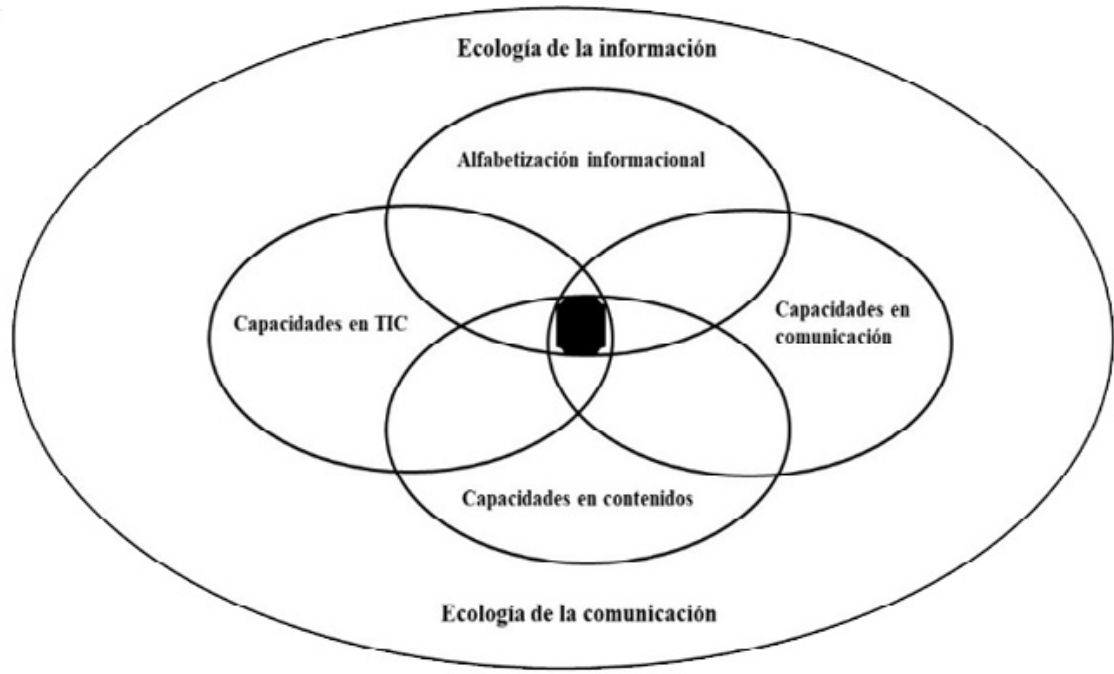

Fuente: Gigler y Bailur, 2014

a) Capacidades en TIC. Se refiere a usar las tecnologías digitales de forma efectiva. Las variables propuestas a medir son: uso del equipo de cómputo, telefonía móvil, tabletas, Smartphone, instalación de aplicaciones, configurar e-mail, trabajar en la nube y manejo de aplicaciones de ofimática.

b) Alfabetización informacional. Pretende encontrar, procesar, evaluar y usar información de una forma efectiva. Las variables que aquí se incluyen son expresadas en forma de pregunta: ¿Para qué busca información?, ¿Con qué fines (personal, laboral, educativa)? ¿Cómo se busca esa información (navegadores, buscadores, etc.)? ¿Cómo sabe que es valiosa o cómo la evalúa la información (criterios)?

c) Capacidades en comunicación. Incluye las formas como el sujeto explora, a través de diversos propósitos, los contenidos y los pone a disposición de otros individuos o grupos usando las TIC. Las variables de este rubro, planteadas en forma de pregunta, son: ¿Con quiénes se comunica a través de las TIC (laboral, familiar y personal), ¿Qué medios utiliza (mensajes, redes sociales, etc.), a través de WhatsApp, Messenger, VolP, otros?

d) Capacidad en contenidos. Comprende la generación de contenidos digitales. Las variables planteadas son: generación de contenido publicado en internet/web, medios de publicación (redes sociales, otros) y objetivo/fin de la publicación (educativo, laboral, personal, etc.).

Cada dimensión se calcula en base a un índice proveniente de ciertas preguntas del instrumento de medición, en donde los índices no se encuentran en base 10 o en base 100. Para determinar cuántos puntos aporta un índice a una dimensión se realizó una regla de tres, considerando que la máxima puntuación por índice corresponde a 2.5 puntos.

\section{Objetivos de la investigación:}

\section{Objetivo general}

Describir las capacidades digitales de estudiantes y docentes de EMS con el propósito de identificar: (i) habilidades para el uso efectivo de las TIC; (ii) niveles de alfabetización informacional; (iii) habilidades de comunicación a través de las tecnologías; y (iv) competencia en la generación de contenidos, todo ello tomando como referencia la aplicación del modelo planteado. 


\section{Objetivos específicos}

a) Comparar comportamientos de estudiantes y docentes universitarios en cuanto a procesos de adaptación en el uso e implementación de diferentes TIC, considerando para ello, las diferencias generacionales en relación con sus capacidades analíticas para la apropiación de las mismas.

b) Identificar en estudiantes y docentes universitarios el grado de uso del conocimiento digital para generar contenidos con aporte a la sociedad y simplificación de la vida con fines educativos.

c) Inferir el grado de empatía hacia las TIC que los estudiantes y docentes de EMS emplean con fines educativos, así como analizar las maneras en que la brecha digital afecta los procesos educativos.

d) Identificar las capacidades de generación de contenidos en los estudiantes y docentes de EMS como parte de sus habilidades digitales.

\section{Resultados}

El análisis se divide en seis apartados: uno relacionado con los datos sociodemográficos y el análisis de cada una de las dimensiones estudiadas, además de un resumen de los resultados globales de las cuatro dimensiones. Los resultados se presentan enseguida:

Datos sociodemográficos. Se caracteriza por lo siguiente:

a) El $69 \%$ de los docentes y estudiantes en estudio corresponden al sexo femenino y $31 \%$ al masculino.

b) El rango de edad de los docentes encuestados es amplio (19.41 años), siendo la edad mínima 38 y la máxima 59, con una Media Aritmética de 40.53 años, en estudiantes la Media Aritmética es de 15.71 años. La diferencia entre estudiantes y docentes es de 40.53 años.

c) El ingreso económico de las familias de los estudiantes es heterogéneo, siendo el mínimo 150 dólares americanos y máximo 1,200 dólares americanos, ambos mensuales. Los docentes observan mayor ingreso familiar, que va de 900 dólares americanos a 1,200 dólares americanos, ambos mensuales.

d) La estructura familiar de los estudiantes se caracteriza como biparental (79.5\%), pero, además, el 16\% manifestó vivir en un hogar formado por madre e hijos y 13.8\% manifiestan vivir solos.

e) El nivel educativo de los estudiantes se asume que la totalidad es de secundaria. Los docentes manifiestan que el $46.6 \%$ tienen licenciatura y el $51.7 \%$ posgrado.

f) El $95.6 \%$ de los encuestados tienen acceso a internet en el hogar; $4.8 \%$ de los estudiantes no tienen y $1.7 \%$ de los docentes igual. La totalidad de los encuestados acceden a la conexión en la institución, tanto en computadoras de la institución como en Tablet que los docentes recibieron como beneficio de trabajo.

g) El $57.4 \%$ de los estudiantes tienen entre 6 a 10 años usando equipo de cómputo (Media Aritmética de 7.01 años). Los docentes manifiestan tener más de 15 años utilizando equipo de cómputo (65.5\%) con una Media Aritmética de 18.53 años y sólo tres casos atípicos sólo con cinco años o menos de experiencia.

h) El promedio de horas de conexión al día en estudiantes es de 8.43 y los docentes 5.69, ambos, usando generalmente el teléfono móvil.

\section{Dimensión 1. Capacidades para el uso efectivo de las TIC}

La capacidad para usar eficientemente las TIC, significa no solamente si se cuenta con conexión a internet, sino que además busca saber en general, las habilidades del usuario y el uso que da a la conexión. Los hallazgos identificados en esta dimensión son los siguientes:

a) El índice de capacidades para el uso efectivo de las TIC según la sumatoria de puntajes de respuestas es 249.88 unidades en estudiantes y 166.72 unidades en docentes, lo que significa una superioridad de los primeros, observándose una diferencia de 83.16.

b) El $91.8 \%$ de los encuestados refieren datos de conexión a computadora, Internet, Tablet y otros dispositivos con una suma total de conexión de 16 horas posibles de conexión al día.

c) Respecto a los objetivos en la búsqueda de información, los estudiantes observan distribución homogénea en porcentajes de uso: $41.79 \%$ de uso educativo/cultural y el $31.37 \%$ lo utilizan con fines laborales o profesionales. En el mismo sentido se observa que el uso familiar y recreativo/ocio es similar entre sí. En el caso de los docentes, la búsqueda de información relacionada con el aspecto laboral/profesional ocupa el primer lugar, con un $50.22 \%$ y el segundo lugar es ocupado por el aspecto educativo/cultural con un $39.47 \%$, cabe señalar que para un docente ambos aspectos están estrechamente relacionados dada la naturaleza de su actividad. El aspecto recreativo/ocio en los docentes presenta una diferencia de treinta puntos porcentuales respecto al de mayor preferencia, que es el laboral/profesional (Figura 2). 
Figura 2. Búsqueda de información por porcentajes en diversos aspectos

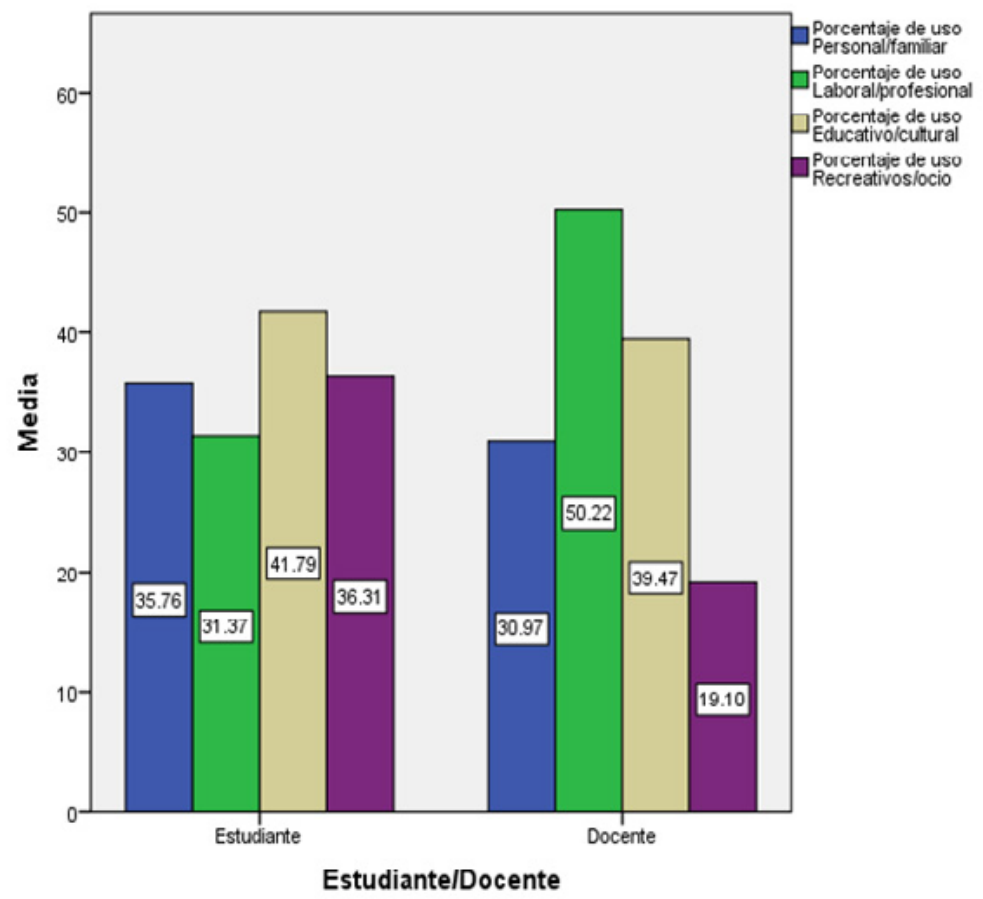

d) El navegador de mayor elección en ambos grupos de estudio es Chrome (348 menciones), segundo lugar Explorer (47 menciones) y tercer lugar Safari (46 menciones).

e) El buscador de mayor elección es Google para ambos grupos de estudio con 376 menciones, en segundo lugar, Yahoo! con 44 elecciones, luego Bing y por último Opera.

f) Tanto las bases de datos como los Metabuscadores ofrecen nulas frecuencias, por lo que se descartan dentro de las capacidades de uso efectivo de las TIC.

g) El uso de los operadores lógicos, el $49.9 \%$ de los estudiantes los utilizan ocasionalmente y en el caso de los docentes el 50.8\% nunca los usan, de la misma manera cuando responden que nunca los usan, donde sí se presentan diferencia sustancial es cuando responden que los utilizan frecuentemente en la que los estudiantes responden en un $\mathbf{8 . 5 0 \%}$ y los docentes el $20.70 \%$ lo cual representa una diferencia de casi 12 unidades porcentuales.

h) Los criterios para buscar y evaluarla importancia de la información son variados, siendo los criterios de orden de la fuente BD, académica, blog y wikis los de mayor relevancia (Tabla 1).

Tabla 1. Criterios para buscar y evaluar la importancia de la información

\begin{tabular}{|l|c|c|}
\hline \multirow{2}{*}{\multicolumn{1}{c|}{ Criterios }} & \multicolumn{2}{c|}{ Menciones } \\
\cline { 2 - 3 } & Estudiantes & Docentes \\
\hline Orden de la fuente BD, académica, blog, wikis & 184 & 35 \\
\hline Orden del buscador u orden de aparición & 166 & 20 \\
\hline Orden del contenido con referencias & 124 & 34 \\
\hline Orden de fecha de publicación & 57 & 19 \\
\hline Orden del autor & 6 & 0 \\
\hline Orden del número de citas & 3 & 5 \\
\hline Orden que sea artículo arbitrado & 0 & 0 \\
\hline
\end{tabular}

\section{Dimensión 2. Capacidades para encontrar, procesar y evaluar información}

Esta dimensión es el resultado del cálculo de la aportación final del diagnóstico de capacidades digitales de acuerdo a los aspectos: personal/familiar, laboral/profesional, educativo/cultural y recreativo/ocio, a través de la sumatoria de las frecuencias válidas para navegadores, buscadores, bases de datos y Metabuscadores mencionados por los encuestados. Los estudiantes obtuvieron 38.74 puntos y los docentes 41.99 puntos, con una diferencia de 3.25 unidades, que es una diferencia muy pequeña a favor de los docentes en el uso de las TIC para encontrar, procesar, evaluar y usar información (Figura 3). 
Figura 3. Capacidades para encontrar, procesar, evaluar y usar información

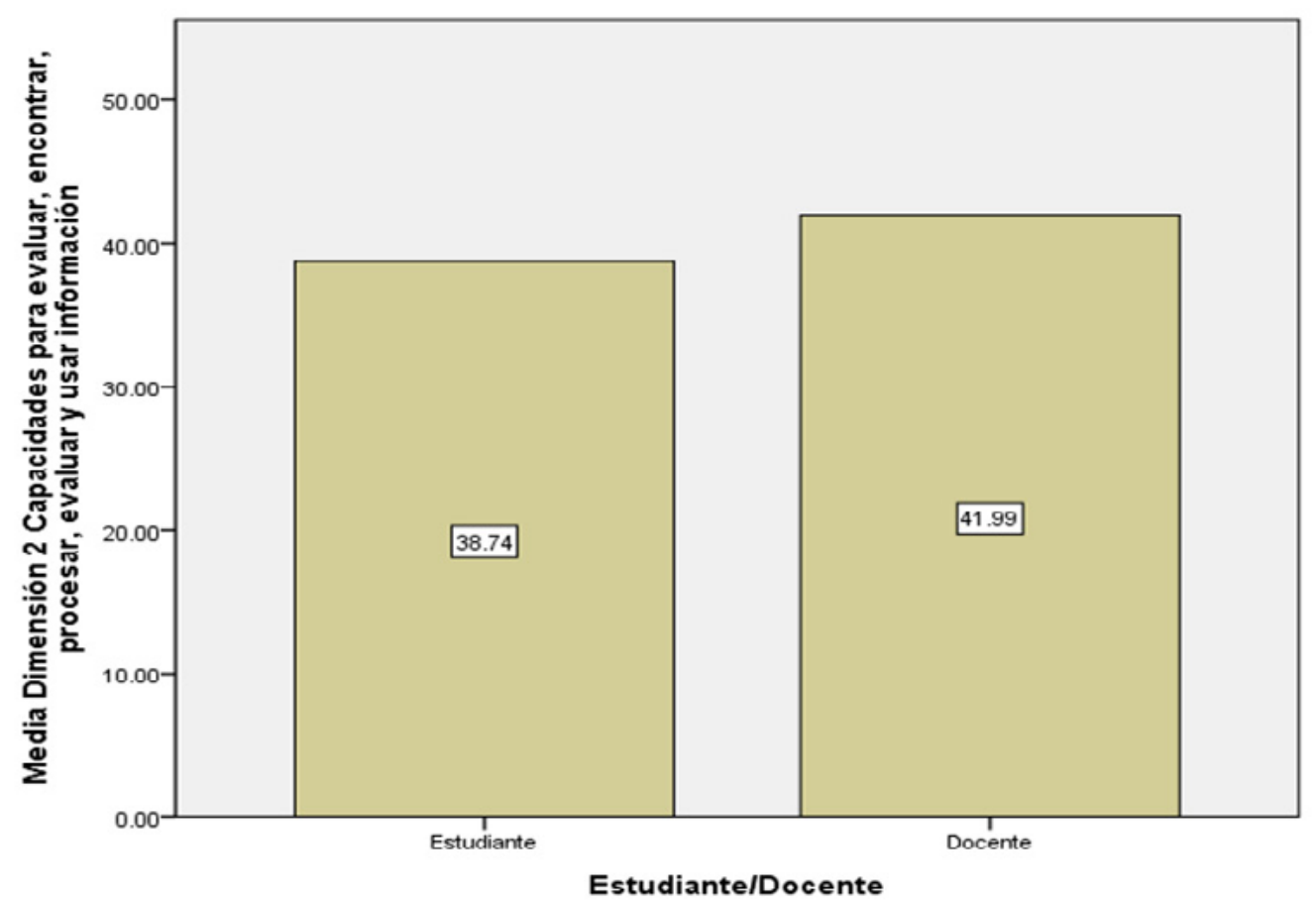

Dimensión 3. Capacidades de comunicación

En esta dimensión se identifican las capacidades de comunicación (cuestionando quién se comunica y qué medios se utilizan). Los resultados de esta dimensión manifiestan lo siguiente:

a) Los estudiantes utilizan las TIC principalmente con fines de comunicación educativa/cultural y personal/familiar presentando medias aritméticas casi similares de 38.95 y 38.87 respectivamente, siendo evidente que el menos utilizado es con fines laboral/profesional $(28.91 \%$ ) y recreativo/ocio $(36.23 \%)$. Por otra parte, en los docentes la mayor elección es la comunicación con fines laboral/profesional, seguido del interés por la comunicación personal/familiar con un $40.02 \%$, en el caso de la comunicación con fines recreativo/ocio, se encuentra una notable diferencia de casi 17 unidades porcentuales por debajo de la más frecuente que es laboral/profesional (Figura 4).

Figura 4. Capacidades de comunicación de estudiantes y docentes

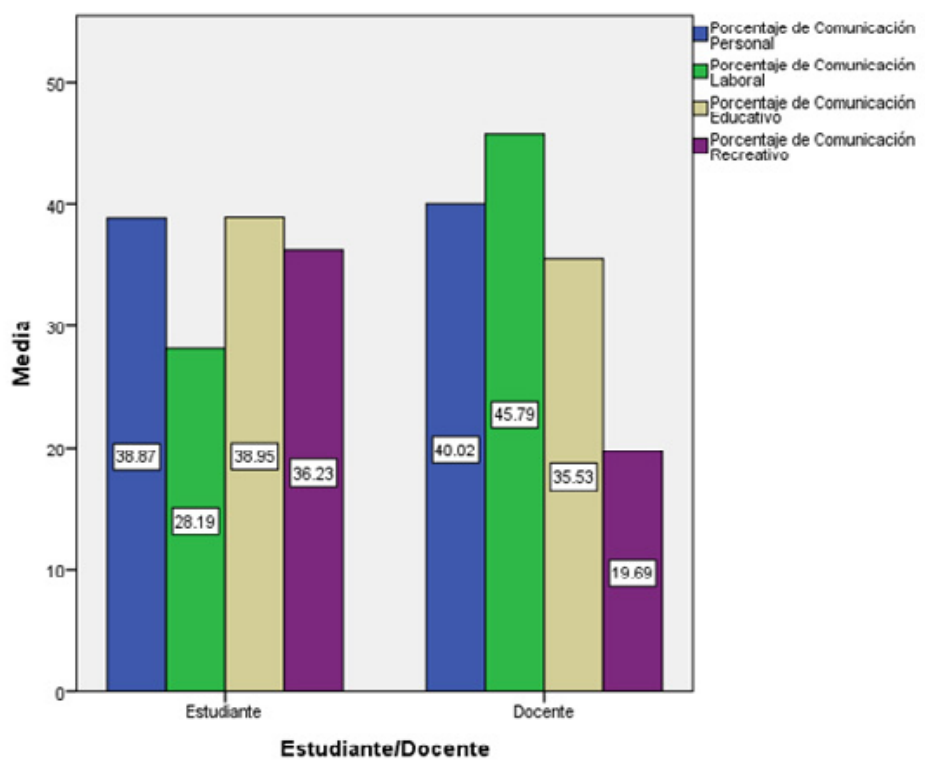


b) WhatsApp es el medio más reconocido para la comunicación personal/familiar (332 menciones), seguido de llamadas telefónicas (199 menciones) y los mensajes SMS (160 menciones), el resto de las opciones se señalan con menor frecuencia (Skype, Facebook, correo electrónico, Twitter, Messenger, Linkedln y Snapchat).

c) En tanto, para el uso laboral profesional se observa al WhatsApp, correo electrónico, llamadas telefónicas y mensajes SMS con las más altas frecuencias.

d) Para el uso educativo/cultural la frecuencia de menciones son: WhatsApp, correo electrónico, mensajes SMS, llamadas telefónicas y Facebook.

e) En el caso de la comunicación con fines recreativo/ocio, se observa que el Facebook desplaza al WhatsApp, las llamadas telefónicas y los mensajes SMS, estos últimos con menores frecuencias.

\section{Dimensión 4. Capacidades para generar contenidos}

Esta dimensión se enfoca a la generación de contenidos para internet, cuestionando a los participantes acerca de los objetivos y formas en el lugar en donde lo han realizado. Los resultados que evalúa esta dimensión son los siguientes:

a) Según los resultados, el $65.6 \%$ de los sujetos en estudio genera contenidos por lo que son consumidores activos; de ellos, los estudiantes superan a los docentes con un $8.2 \%$, siendo estos últimos los que publican contenido sólo en $58.6 \%$, en tanto los estudiantes son el $66.8 \%$.

b) Se observan variaciones respecto a los motivos de publicación de contenidos, siendo el objetivo principal de los estudiantes el educativo/cultural y la más baja frecuencia se encontró en el laboral/profesional. De los docentes sobresale en este aspecto el contenido generado con el objetivo laboral/profesional con $34.83 \%$, observándose en último lugar el aspecto recreativo/ocio con una diferencia de 20 puntos porcentuales respecto al de objetivo laboral/profesional (Figura 5).

Figura 5. . Porcentaje de publicación de contenidos por alumnos y docentes

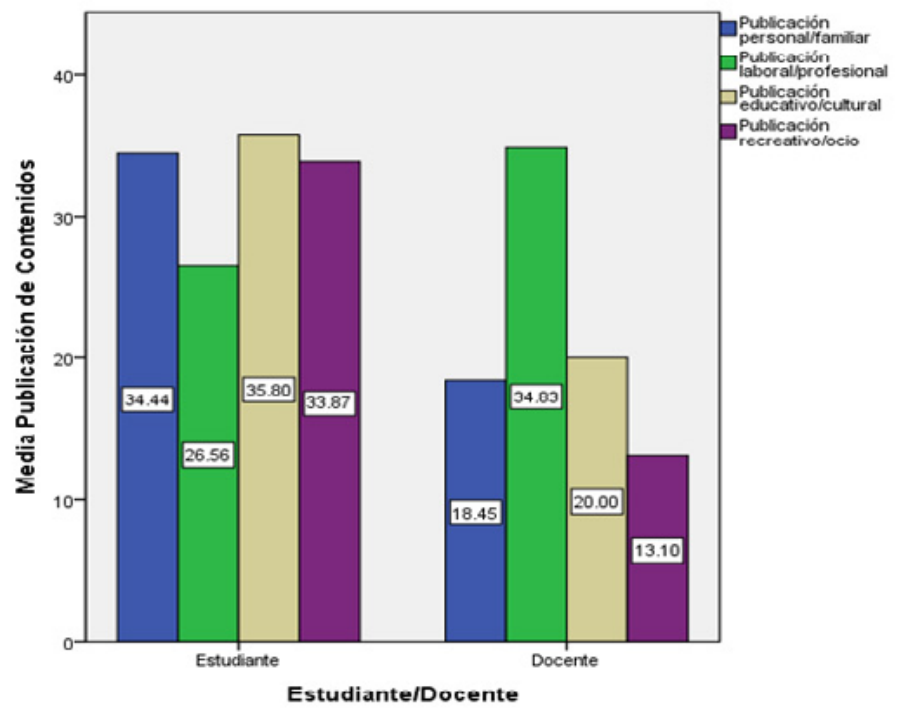

c) La tendencia de los estudiantes a publicar artículos en línea, colaboraciones y wikis con objetivo educativo/ cultural es del $37.2 \%$, desplazando a los fines recreativo/ocio y personal/familiar por casi 25 unidades porcentuales, así mismo el porcentaje de quienes no hacen este tipo de publicaciones corresponde a un $30.2 \%$. En el caso de los docentes destaca en primer lugar quienes no han publicado artículos en línea, colaboraciones y wikis con un 48.3\%, seguido de los objetivos laboral/profesional con un 32.8\%, y educativo cultural con un 15.5 $\%$, sólo un docente respondió que publica artículos en línea, colaboraciones y wikis con fin recreativo o de ocio y uno más respondió que su objetivo es personal/familiar.

d) El $42.9 \%$ de los participantes en el estudio no han publicado talleres, cursos y material didáctico, situación en la que coinciden estudiantes y docentes; sin embargo, en los que sí han publicado sobresalen los objetivos laborales/profesionales para los docentes con un 34.5\% y para los estudiantes el primer lugar es el educativo/ cultural con un $28.40 \%$ de los encuestados.

e) Además, en la Tabla 23 se aprecia que el $41.1 \%$ de la población encuestada no ha publicado videos y animaciones, coinciden en este rubro docentes y estudiantes; sin embargo los estudiantes señalan en un $26.9 \%$ que sus publicaciones son con objetivos recreativos o de ocio como primer lugar de los que sí publican videos o animaciones, y en segundo espacio de los que sí publican el educativo/cultural con un $18.4 \%$ mientras los docentes destacan que el $15.5 \%$ tienen como objetivo el laboral/profesional, y en última instancia el personal 
familiar con un $3.4 \%$ de las encuestas de docentes.

f) El $33.2 \%$ de los estudiantes manifiestan no publicar presentaciones ni slideshows. Destaca en primer lugar que el $49.2 \%$ de los estudiantes encuestados publican presentaciones y slideshows con fin educativo/cultural, así como el $10.6 \%$ con objetivo laboral/profesional; los docentes por otra parte publican en un $29.3 \%$ con el objetivo laboral/profesional y un $13.8 \%$ con fines educativo/cultural y el $55.2 \%$ no publican presentaciones ni slideshows.

g) Respecto al reconocimiento de los encuestados sobre alguna habilidad especial en el uso de las TIC o sus necesidades de adquisición de otros conocimientos específicos, la lista de propuestas fue variada y abundante, principalmente aquellas relacionadas con el uso de paquetes computacionales básicos, trabajar en la nube, programación computacional, aprender a utilizar impresoras. En general las temáticas fueron vinculadas a temáticas básicas.

\section{Análisis global de las cuatro dimensiones}

A partir de los resultados de cada dimensión, se estableció una ponderación para asignar una calificación máxima por dimensión, así mismo se realizó un análisis de los denominados datos reales, mismos que corresponden únicamente a aquellas encuestas cuyos datos presentan correspondencia aritmética de acuerdo a las instrucciones indicadas en el instrumento de recolección de datos. Producto de lo anterior se logró establecer una escala de interpretación de los valores ponderados de las cuatro dimensiones, de tal forma que se transforma la variable cuantitativa en cualitativa asignando puntuaciones de acuerdo a una escala de interpretación (Tabla 2).

Tabla 2. Escala de interpretación

\begin{tabular}{|c|c|}
\hline Promedio & Interpretación \\
\hline $0-1.99$ & Muy bajo \\
\hline $2.00-3.99$ & Bajo \\
\hline $4.00-5.99$ & Medio \\
\hline $6.00-7.99$ & Alto \\
\hline $8.00-10$ & Muy alto \\
\hline
\end{tabular}

En la escala de 10 puntos calculada a partir de las cuatro dimensiones, se observa que en promedio los docentes obtienen 3.08 mientras que los estudiantes presentan un valor de 3.18 lo cual hace una diferencia de 0.10 unidades. Por tanto, los resultados observados son: $61.3 \%$ de los datos se concentran en un nivel bajo, en tanto, sólo el $1.3 \%$ de los mismos alcanza el nivel muy alto.

Respecto a las Medidas de Tendencia Central de la ponderación de las cuatro dimensiones, se observa una Media Aritmética de 3.16 (promedio de interpretación bajo), con un Mínimo de 0.22 y un Máximo de 8.88, lo cual da como resultado una Desviación Estándar igual a 1.31 unidades. En congruencia con el análisis global de las cuatro dimensiones, en el histograma de distribución de frecuencias se observan todos los datos desplazados hacia la izquierda de la gráfica en un nivel bajo en este trabajo diagnóstico (Figura 6).

Figura 6. Histograma de distribución de frecuencias

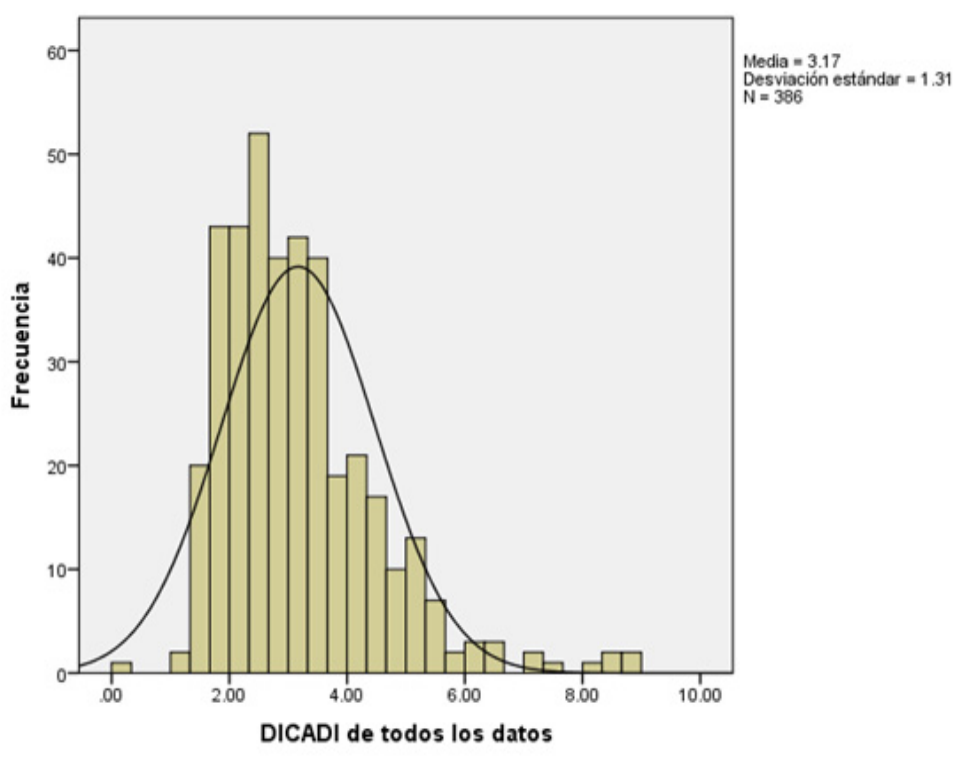


Además, se realizó una prueba $\mathrm{Q}-\mathrm{Q}$ Normal de las cuatro dimensiones, en donde se observan valores que se encuentran fuera de la línea del valor normal esperado, lo cual tiene relación con aquellas respuestas obtenidas en preguntas que no tienen correspondencia aritmética, considerando la instrucción indicada en la pregunta (Figura 7).

Figura 7. Q-Q Normal de DICADI de todos los datos

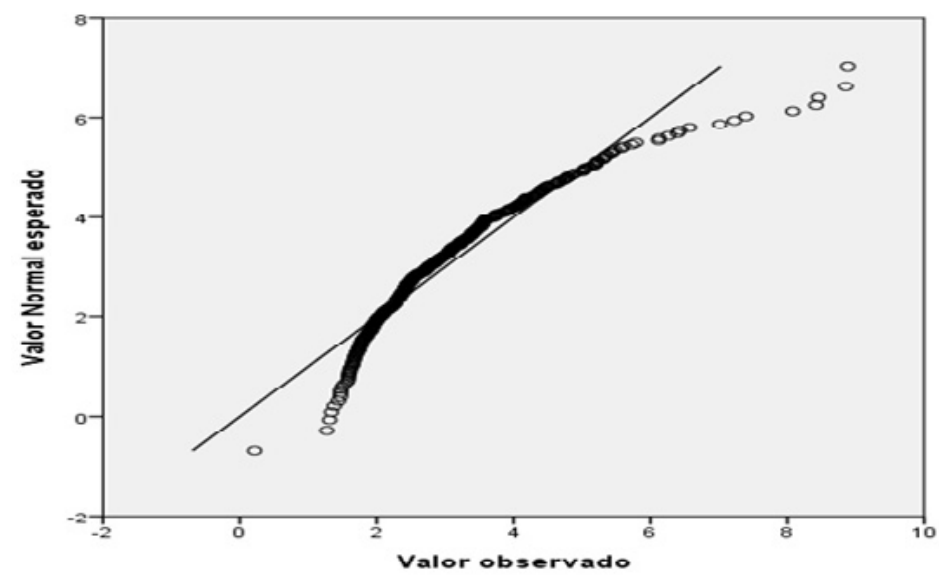

Para continuar en el análisis de inconsistencias aritméticas, se realizó un comparativo entre las Medias Aritméticas de los resultados de las cuatro dimensiones analizadas en este estudio, de lo cual se obtiene como resultado global 3.1684, parámetro traducido a nivel cualitativo que corresponde al nivel bajo de capacidades digitales. Así mismo, se realizó un análisis de datos cuyas respuestas son aritméticamente correctas, a lo que se determina como datos reales de las cuatro dimensiones, de lo cual se obtiene una Media Aritmética para la población estudiada igual a 5.9708 (en variable cualitativa equivale a un nivel medio de capacidades digitales), situación que no es concluyente ya que los valores reales corresponden al $50.25 \%$ del total.

\section{Conclusiones}

La era digital es tan reciente, que plantear una metodología para determinar las capacidades digitales de las personas implica grandes retos, ya que los diagnósticos previos son pocos y las variantes muchas. Cuando se trabaja con percepciones, gran parte de las investigaciones en cualquier nivel educativo dependen de la sinceridad y buen juicio del encuestado. Esta investigación no es la excepción y sus resultados pueden presentar errores debido a inconsistencias en las respuestas por parte de los entrevistados, mismas que podrían presentarse de forma consciente e intencional al exagerar sobre las propias capacidades individuales o de forma inconsciente al no comprender las instrucciones de las preguntas o al omitir leerlas, lo cual representa la principal debilidad de este estudio.

Los resultados obtenidos en esta investigación arrojaron una calificación ligeramente superior en los docentes debido a que el diagnóstico no se basa solamente en las horas de conexión, sino en la capacidad de utilizar ese tiempo para realizar actividades productivas. Esta información es reveladora pues esa diferencia se presentó a pesar de la brecha existente en las condiciones sociodemográficas como nivel de estudios, ingreso familiar y sobre todo la edad (con un rango de diferencia de 20 años entre ambos grupos). Por lo anterior es importante señalar la necesidad de establecer un monitoreo periódico de los nuevos usuarios con el fin de determinar en qué medida aprovechan las bondades tecnológicas en aspectos productivos y no solamente en cuestiones lúdicas. Esto es importante porque las capacidades digitales son determinantes al momento de establecer indicadores mundiales y su desarrollo impacta positivamente en la competitividad del país.

Se ofrecen a continuación algunas conclusiones específicas:

a) Aplicando el modelo planteado, considerando las cuatro dimensiones se obtiene una calificación para la población en estudio igual a 3.1684; para estudiantes se obtiene un 3.18 y para docentes un 3.08, cifras que no se consideran significativas entre ambas generaciones; traduciendo estos datos a un valor cualitativo se concluye que tanto estudiantes como docentes presentan un nivel bajo en el desarrollo de capacidades digitales de estudiantes y docentes de EMS.

b) Respecto a medición de capacidades para usar las TIC, se observó un valor promedio de 0.6184 de un máximo posible igual a 2.5, en el caso de los estudiantes se observa que obtienen un índice de 0.6507, valor mayor en un $33 \%$ que los docentes quienes obtuvieron 0.4342 , con lo que se comprueba que los estudiantes presentan mayores índices en el uso de las TIC. 
c) Al analizar la Dimensión 2, se obtiene que los docentes buscan información con objetivos laboral/profesional y educativo/cultural en un $89.69 \%$ y 19.10 busca el aspecto recreativo/ocio, mientras que los estudiantes buscan información en un $73.16 \%$ de los aspectos laboral/profesional y educativo/cultural y $36.31 \%$ del aspecto recreativo/ocio.

d) En cuanto al grado de uso del conocimiento digital para generar contenidos con aporte a la sociedad y simplificación de la vida con fines educativos, los estudiantes obtienen 0.9224 de un máximo de 2.5 y los docentes 0.9998 unidades, si bien en la Dimensión 1 se observa que los estudiantes utilizan más las TIC, los docentes obtienen un mejor índice para encontrar, procesar, evaluar y usar información. El 73.16\% de la población de estudiantes y el $89.69 \%$ de docentes busca información relacionada con actividades laboral/profesional y educativo/cultural.

e) El grado de empatía por estudiantes y docentes de EMS hacia las TIC con fines educativos, buscando observar las capacidades de comunicación, se obtiene un índice global de 1.0029 de un máximo posible de 2.5; en el cual los estudiantes obtienen un valor de 0.9873 y los docentes un 1.0922 , de lo cual se obtiene que los estudiantes utilizan las TIC para comunicarse en los cuatro aspectos analizados de manera homogénea. No así en los docentes en donde se mayor variabilidad: el porcentaje mayor corresponde a la comunicación mediante TIC de 45.79 del aspecto laboral/profesional, el educativo/cultural en un 35.53, dejando al recreativo/ocio en un 19.69, lo cual indica que los estudiantes han incluido las TIC en los diversos aspectos de su vida.

f) Las capacidades para generar contenidos arrojan como resultado que el $65.60 \%$ de la población estudiada y el $58.60 \%$ los docentes si ha publicado contenidos (artículos, wikis y colaboraciones), así como talleres, cursos y material didáctico que representan más del 30 por ciento de los fines de publicación.

El vertiginoso avance del conocimiento no es un factor que escape al DICADI. Para lograr mantener actualizada una herramienta tan importante como esta es necesario adecuar constantemente los factores que evalúa, pero respetando siempre las cuatro dimensiones que se plantean para lograr obtener puntuaciones que eviten en la medida de lo posible la pérdida de la objetividad y la validez de las mediciones. Estos importantes avances se podrían presentar en el marco de una visión más holística de la alfabetización informacional tomando en cuenta nuevas herramientas digitales como las aulas invertidas, el aprendizaje experiencial, los espacios de creación y la colaboración entre los bibliotecarios, informáticos y docentes para la facilitación del uso y aprendizaje de los recursos de hardware y software.

\section{Referencias}

AGUADED-GÓMEZ, Ignacio. Miopía en los nuevos planes de formación de maestros en España: ¿docentes analógicos o digitales? Comunicar [online]. 2009, vol. 33, n.XVII, pp. 7-8. Disponible en https://www.revistacomunicar. com/numeros anteriores/archivospdf/33/c33-2009-00-001.pdf. ISSN 1988-3293. DOI:10.3916/c33-2009-00-001

AGUIAR, Brunell; VELÁZQUEZ, René y AGUILAR, Jorge. Innovación docente y empleo de las TIC en la educación superior. Revista Espacios [online]. 2019, vol. 40, n. 2, pp. 1-12. Disponible en https://www.revistaespacios. com/a19v40n02/a19v40n02p08.pdf. ISSN 07981015.

ANTÚNEZ SÁNCHEZ, Armando Guillermo; MORALES SALAS, Rubí Estela y ORTIZ ORTIZ, María Gloria. Infotecnología: una cultura ineludible en el docente de la educación superior del siglo XXI. Opuntia Brava: Revista Electrónica [online]. 2019, n. 11 (Especial), pp. 171-181. Disponible en http://opuntiabrava.ult.edu.cu/index.php/ opuntiabrava/article/view/675. ISSN 2222-081X.

CASTAÑEDA, Linda; ESTEVE, Francesc y ADELL, Jordi. ¿Por qué es necesario repensar la competencia docente para el mundo digital? RED. Revista de Educación a Distancia [online]. 2018, vol. 56, n. 6, 1-20. ISSN 1578-7680. DOI: http://dx.doi.org/10.6018/red/56/6

CEBALLOS, Francisco. México Forbes [online]. 2013. Disponible en http://www.forbes.com.mx/educaciontecno$\underline{\text { logical }}$

COLÁS BRAVO, María del Pilar; DE PABLOS PONS, Juan y BALLESTA PAGÁN, Javier. Incidencias de las TIC en la enseñanza en el sistema educativo español: una revisión de la investigación. RED. Revista de Educación a Distancia [online]. 2018, vol. 52, n. 2, pp. 1-23. ISSN 1578-7680. DOI: http://dx.doi.org/10.6018/red/56/2 http:// www.um.es/ead/red/56/colas et al.pdf

DEL CASTILLO-ALEMÁN, Gloria. Las políticas educativas en México desde una perspectiva de política pública: gobernabilidad y gobernanza. Magis, Revista Internacional de Investigación en Educación [online]. 2012, vol. 4, n. 9, pp. 637-652. Disponible en http://magisinvestigacioneducacion.javeriana.edu.co/. ISSN 2027-1174. 
DURÁN CUATRERO, Marta; PRENDES ESPINOSA, Ma Paz y GUTIÉRREZ PORLÁN, Isabel. Certificación de la Competencia Digital Docente: propuesta para el profesorado universitario. RIED. Revista Iberoamericana de Educación a Distancia [online]. 2019, vol. 22, n. 1, pp. 187-205. Disponible en http://revistas.uned.es/index.php/ ried/article/view/22069. ISSN 1390-3306. DOI: http://dx.doi.org/10.5944/ried.22.1.22069

FERNÁNDEZ-CRUZ, Francisco José y FERNÁNDEZ-DÍAZ, Ma José. Los docentes de la Generación Z y sus competencias digitales. Comunicar [online]. 2016, vol. 46, n. 24, pp. 97-105. Disponible en file:///C:/Users/User/ Downloads/10.3916_C46-2016-10\%20(3).pdf. ISSN 1988-3293. DOI http://dx.doi.org/10.3916/C46-2016-10.

GIGLER, Björn-Sören. Informational Capabilities: The Missing Link for the Impact of ICT on Development. E-Transform Knowledge Platform Working Paper [online]. Washington, D.C.: World Bank, 2011. Disponible en https:// openknowledge.worldbank.org/handle/10986/19011

GIGLER, Björn-Sören y BAILUR, Savita. Closing the Feedback Loop Can Technology Bridge the Accountability Gap? [online]. 2014. Disponible en https://openknowledge.worldbank.org/bitstream/handle/10986/ ISBN: 978-14648-0192-1

GÓMEZ COLLADO, Martha. Panorama del sistema educativo mexicano desde la perspectiva de las políticas públicas. Innov. educ. [online]. 2017, vol.17, n.74, pp.143-163. Disponible en http://www.scielo.org.mx/pdf/ie/ v17n74/1665-2673-ie-17-74-00143.pdf. ISSN 1665-2673.

GONZÁLEZ-QUIÑONES, Fidel; TARANGO, Javier y FIERRO-RAMÍREZ, Luis Alberto. Identificación de capacidades digitales en estudiantes y docentes universitarios del área de humanidades. Cuadernos de Documentación Multimedia [online]. 2018, vol. 29, n. 1, pp. 54-78. Disponible en https://revistas.ucm.es/index.php/CDMU/issue/ view/3231/showToc. ISSN-e 1575-9733. http://dx.doi.org/10.5209/CDMU.60542

GONZÁLEZ QUIÑONES, Fidel; TARANGO, Javier y VILLANUEVA-LEDEZMA, A. Hacia una propuesta para medir capacidades digitales en usuarios de internet. Revista Interamericana de Bibliotecología [online]. 2019, vol. 42, n. 3, 197-212. ISSN-e 2538-9866. http://dx.doi.org/10.17533/udea.rib. v42n3a01

HERNÁNDEZ RAMOS, Juan Pablo y TORRIJOS FINCIAS, Patricia. Percepción del profesorado sobre la integración de las Tecnologías de la Información y la Comunicación (TIC) en las modalidades docentes. Influencia del género y la edad. EDMETIC, Revista de Educación Mediática y TIC [online]. 2018, vol. 8, n. 1, pp. 128-146. Disponible en file:///C:/Users/User/Downloads/10.3916_C46-2016-10\%20(3).pdf. ISSN 2254-0059. DOI: https:// doi.org/10.21071/edmetic.v8i1.10537.

LÓPEZ, Karina. La educación y la tecnología: una educación simbiótica [online]. 2012, Disponible en https://marketingeducativo.mx/2012/04/20/la-educacion-y-la-tecnologia-una-relacion-simbiotica/

LÓPEZ MENESES, Eloy y ESTEBAN IBÁÑEZ, Macarena. La educación social y las nuevas tecnologías de la información y la comunicación: nuevos espacios en la construcción e intervención socioeducativa. Revista Latinoamericana de Estudios Educativos [online]. 2008, vol. XXXVIII, n. 1-2, pp. 255-287. Disponible en: http://www. redalyc. org/articulo. oa? id=27012437010. ISSN: 0185-1284.

MARCELO, Carlos. Las tecnologías para la innovación y la práctica docente. Revista Brasileira de Educação [online]. 2013, vol. 18, n. 52, 25-47. Disponible en http://www.scielo.br/pdf/rbedu/v18n52/03.pdf. ISSN 14-13-2478. http://dx.doi.org/10.1590/S1413-24782013000100003

MCGINN, Noel; RIVERA, Eduardo y CASTELLANOS, Adrián. El Sistema Educativo Mexicano (un modelo de simulación de escenarios). Revista Latinoamericana de Estudios Educativos [online]. 2014, vol. XLIV, n. 2, pp. 143-185. Disponible en http://www.redalyc.org/articulo.oa?id=27031268006. ISSN: 0185-1284.

MORENO RODRÍGUEZ, María Dolares; GABARDA MÉNDEZ, Vicente y RODRÍGUEZ MARTíN, Ana. Alfabetismo informacional y competencia digital en estudiantes de magisterio. Profesorado: Revista de Curriculum y Formación del Profesorado [online]. 2018, vol. 22, n. 3, pp.1-18. Disponible en http://digibug.ugr.es/handle/10481/53393. ISSN-e: 1138-414X. DOI: 10.30827/profesorado.v22i3.8001

PAREDES-PARADA, Wladimir. Brecha en el uso de tecnologías de la información y comunicación (TIC) básicas y modernas entre estudiantes y docentes en universidades ecuatorianas. Revista Educación [online]. 2019, vol. 43, n. 1, pp. 1-18. Disponible en https://revistas.ucr.ac.cr/index.php/educacion/article/view/27423. ISSN: 0379-7082. 
DOI: https://doi.org/10.15517/revedu.v43i1.27423

RAMÍREZ RAYMUNDO, Rodolfo. El cambio educativo necesario: los desafíos de la política. En La política educativa del sexenio 2013-2018: alcances y límites (pp. 9-16). Ciudad de México: Universidad Iberoamericana, 2015.

TEJEDA FERNÁNDEZ, José y POZOS PÉREZ, Katia. Nuevos escenarios y competencias digitales docentes: hacia la profesionalización docente con TIC. Profesorado: Revista de Curriculum y Formación del Profesorado, 2018, vol. 22, n. 1, pp. 25-51. Disponible en https://recyt.fecyt.es/index.php/profesorado/article/view/63620/0. ISSN 1138-414X.

UGALDE SÁNCHEZ, Cecilia Esperanza y GONZÁLEZ CABRERA, Catalina del Rosario. Los jóvenes y su participación en los medios digitales. En Zacipa-Infante, I., Tur-Viñes, V., Segarra-Saavedra, J. (Coord.). Tendencias publicitarias en Iberoamérica: diálogos de saberes y experiencias [online]. (pp. 367-378). Alicante, España: Universidad de Alicante, 2016. ISBN: 978-84608-3444-1. DOI:10.14198/MEDCOM/2016/8

VALENCIA-ALTAMIRANO, Juan Carlos; TOPÓN GUALOTUÑA, Diego Rafael. y PÉREZ FABAR, María Augusta. El analfabetismo digital en docentes limita la utilización de los EVEA. Revista Publicando, 2016, vol. 3, n. 8, pp. 24-36. Disponible en https://dialnet.unirioja.es/descarga/articulo/5833406.pdf. ISSN: 1390-9304.

VIÑALS BLANCO, Ana y CUENCA AMIGO, Jaime. El rol del docente en la era digital. Revista Interuniversitaria de Formación del Profesorado [online]. 2016, vol. 30, n. 2, pp. 103-114. Disponible en https://www.redalyc.org/ pdf/274/27447325008.pdf. ISSN 0213-8646.

WEISS HORZ, Eduardo. Los significados del bachillerato para los jóvenes y la permanencia escolar en México. Sinéctica: Revista Electrónica de Educación [online]. 2016, vol. 30, n. 2, pp. 103-114. ISSN 2007-7033. DOI: 10.31391/S2007-7033(2018)0051-003

ZAVALA, Diego; MUÑOZ, Karlita y LOZANO, Edwin. Un enfoque de las competencias digitales de los docentes. Revista Publicando [online]. 2016, vol. 3, n. 9, pp. 330-334. Disponible en: https://revistapublicando.org/revistal index.php/crv/article/view/353. ISSN 1390-9304.

ZEMPOALTECA DURÁN, Beatriz; BARRAGÁN LÓPEZ, Jorge Francisco; GONZÁLEZ MARTíNEZ, Juan y GUZMÁN FLORES, Teresa. Formación en TIC y competencia digital en la docencia en instituciones públicas de educación superior. Apert. (Guadalaj., Jal.) [online]. 2017, vol.9, n.1, pp.80-96. ISSN 2007-1094. http://dx.doi. org/10.18381/Ap.v9n1.922. 


\section{Dados dos autores}

Javier Tarango

Miembro del Sistema Nacional de Investigadores (Nivel II). Doctorado en Educación por la Universidad Autónoma de Chihuahua, México (UACH); Maestría en Ciencias de la Información (Universidad de Guanajuato, México) y en Desarrollo Organizacional (Universidad de Monterrey, México). Labora como Profesor- Investigador de Tiempo Completo en la UACH desde 1996 en los programas académicos de Maestría en Innovación Educativa y Doctorado en Educación, Humanidades y Arte (ambos programas reconocidos por el Programa Nacional de Posgrados de Calidad - PNPC); además imparte cátedra virtual en la Licenciatura en Bibliotecología y Gestión del Conocimiento, y en la Maestría en Transparencia y Protección de Datos Personales (reconocido por el PNPC) de la Universidad de Guadalajara. Ha sido presidente del comité organizador de los siguientes congresos internacionales: Transborder Library Forum, 2005; Jornadas Mexicanas de Biblioteconomía, 2008; Trejo Foster Foundation Institute, 2012; Simposium Internacional de Educación Media Superior, 2013; Conferencia Internacional sobre Brecha Digital e Inclusión Social, 2014; Congreso Internacional de Información, Comunicación e Investigación, 2017 y 2018; Seminario de Información y Sociedad de la Universidad Nacional Autónoma de México, Instituto de Investigaciones Bibliotecológicas y de la Información UNAM-IIBI, 2018. Miembro del Instituto Agustín Millares Carlo de Documentación y Gestión de Información de la Universidad Carlos III de Madrid y del Seminario de Información y Sociedad (UNAM-IIBI). Líder del Cuerpo Académico Consolidado UACH-088 'Estudios de la Información', sus líneas de investigación se relacionan con la evaluación de la producción y comunicación científica; gestión del conocimiento y la innovación; e identificación y desarrollo de la cultura informacional. Es árbitro de más de 30 revistas científicas internacionales.

jtarango@uach.mx

\section{Fidel González-Quiñones}

Miembro del Sistema Nacional de Investigadores (SNI) Es profesor investigador de tiempo completo en la Facultad de Filosofía y Letras de la Universidad Autónoma de Chihuahua (UACH) en los niveles de licenciatura, maestría y doctorado. Tiene una licenciatura en Ciencias de la Información, un MBA en Recursos Humanos y otro en Marketing además de un doctorado en Periodismo Social por la Universidad de Sevilla. Es un analista de datos con más de 15 años de experiencia como conferencista e investigador y actualmente dirige el Centro Estratégico de Investigación de la $\mathrm{UACH}$, que desarrolla estudios interdisciplinarios con particulares, empresas y entidades de gobierno estatal y federal. Miembro del Cuerpo Académico Consolidado UACH-CA-088 Estudios de la Información. Autor de diversas publicaciones en revistas indexadas.

fgonzalez@uach.mx

\section{Elsa Ivonne Morales-Ángel}

Máster en Ingeniería en Vías Terrestres y Máster en Educación Superior (ambas en la Universidad Autónoma de Chihuahua, México). Es profesora del área de Química en el Colegio de Bachilleres del Estado de Chihuahua, México, desde hace 20 años.

elsa.morales@cobachih.edu.mx

Received: 2019-03-18

Accepted: 2020-10-24

\section{(c) $)$ EY}

This work is licensed under a Creative Commons Attribution 4.0 United States License.

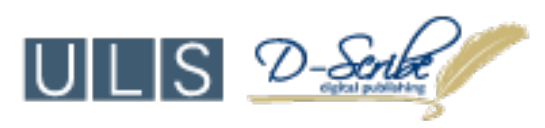

This journal is published by the University Library System of the University of Pittsburgh as part of its D-Scribe Digital Publishing Program and is cosponsored by the University of Pittsburgh Press 